\title{
Directional shift current in mirror-symmetric $\mathrm{BC}_{2} \mathbf{N}$
}

\author{
Julen Ibañez-Azpiroz $\odot,{ }^{1}$ Ivo Souza $\odot,{ }^{1,2}$ and Fernando de Juan $\odot^{2,3}$ \\ ${ }^{1}$ Centro de Física de Materiales, Universidad del País Vasco, EHU, 20018 San Sebastián, Spain \\ ${ }^{2}$ Ikerbasque Foundation, 48013 Bilbao, Spain \\ ${ }^{3}$ Donostia International Physics Center, 20018 San Sebastián, Spain
}

(Received 26 June 2019; revised manuscript received 16 September 2019; accepted 13 February 2020; published 5 March 2020)

\begin{abstract}
We present a theoretical study of the shift current in a noncentrosymmetric polytype of graphitic $\mathrm{BC}_{2} \mathrm{~N}$. We find that the photoconductivity near the fundamental gap is strongly anisotropic due to the vanishing of particular tensor components not foretold by point-group symmetry arguments; this is a consequence of dipole selection rules imposed by mirror symmetry, which imply that the relative parities between valence and conduction bands are key for determining the directionality of the band-edge response. In addition, the band-edge photoconductivity turns out to be rather large, with the peak value occurring in an energy range suitable for optical manipulation.
\end{abstract}

DOI: 10.1103/PhysRevResearch.2.013263

\section{INTRODUCTION}

The conversion of light into electrical current via the photovoltaic effect is one of the most copious supplies of renewable energy on earth. Traditional solar cells make use of $p$ - $n$ junctions to generate a built-in electric field that drives the photoexcited electrons. It has long been known that single-phase noncentrosymmetric crystals exhibit a different type of photovoltaic effect, called the bulk photovoltaic effect (BPVE) [1-3]. This is a nonlinear optical response that consists in the generation of a photovoltage or photocurrent upon light absorption. In recent years, the study of the BPVE has been reinvigorated by the search for novel materials with large photoresponsivities [4-8], as well as by its sensitivity to geometric and topological properties of the electronic wave functions [9-11]. As an example, the sign of the shift-current contribution to the BPVE has been suggested as a possible probe for detecting topological phase transitions [12,13]. In addition, recent experimental work on TaAs found large and anisotropic contributions to the shift-current BPVE driven by the low-energy Weyl-node physics [14].

In this work we report a distinctive shift-current response at the band edge of a noncentrosymmetric polytype of graphitic $\mathrm{BC}_{2} \mathrm{~N}$, a layered semiconductor made of alternating zigzag chains of carbon and boron nitride [15-21]. Our $a b$ initio calculations show that near the fundamental band gap the calculated response exhibits strong anisotropy, due to the vanishing of certain tensor components not foretold by phenomenological symmetry arguments. We trace the origin of this anisotropy to the mirror symmetry of the crystal, which

Published by the American Physical Society under the terms of the Creative Commons Attribution 4.0 International license. Further distribution of this work must maintain attribution to the author(s) and the published article's title, journal citation, and DOI. imposes selection rules on dipole transitions between the band edges. We capture the essential physics of this phenomenon with a two-band $\mathbf{k} \cdot \mathbf{p}$ model, thus providing a suitable framework for a broad class of materials.

\section{SHIFT-CURRENT BPVE}

The BPVE is a nonlinear optical response of the form [1]

$$
J_{a}=\sum_{b, c} \sigma^{a b c}(\omega) E_{b}(\omega) E_{c}(-\omega),
$$

where $a, b$, and $c$ are Cartesian indices. Since both the electric field $\mathbf{E}$ and the current $\mathbf{J}$ are odd under inversion, the BPVE can only occur in systems where this symmetry is broken. The right-hand side of Eq. (1) can be split into symmetric and antisymmetric parts under $b \leftrightarrow c$, known, respectively, as the linear and circular BPVEs [1]; the former occurs in piezoelectric crystals and the latter in gyrotropic crystals. The shift current is the intrinsic (interband) contribution to the linear BPVE.

In the independent-particle picture, the shift photoconductivity takes the form [22]

$$
\sigma^{a b c}(\omega)=C \int \frac{d^{3} k}{(2 \pi)^{3}} \sum_{n, m} f_{n m} I_{m n}^{a b c} \delta\left(\omega_{n m}-\omega\right),
$$

where $C=-\pi g_{s}|e|^{3} / 2 \hbar^{2}$ is a combination of fundamental constants ( $g_{s}=2$ accounts for spin degeneracy), $f_{n m}=f_{n}-$ $f_{m}$ and $\hbar \omega_{n m}=E_{n}-E_{m}$ are differences in band occupations and energies, and the matrix element reads

$$
I_{m n}^{a b c}=\operatorname{Im}\left(r_{m n}^{b} r_{n m}^{c ; a}\right)+b \leftrightarrow c .
$$

Here $r_{m n}^{b}$ is the interband dipole (the off-diagonal part of the Berry connection matrix $\left.A_{m n}^{b}=i\left\langle u_{m \mathbf{k}} \mid \partial_{k_{b}} u_{n \mathbf{k}}\right\rangle\right)$, which only depends on the initial and final states $m$ and $n$, and $r_{n m}^{c ; a}$ is a generalized derivative defined as $r_{n m}^{c ; a}=\partial_{k_{a}} r_{n m}^{c}-i\left(A_{n n}^{a}-\right.$ 
$\left.A_{m m}^{a}\right) r_{n m}^{c}$, which depends implicitly on intermediate virtual states [22].

\section{MIRROR SYMMETRY AND THE ROLE OF BAND-EDGE PARITIES}

We next analyze the constraints imposed on the shift photoconductivity tensor by the presence of mirror symmetry; without loss of generality, we choose $M_{x}: x \rightarrow-x$.

Let us start with the conventional symmetry analysis of response tensors [23,24]. If $M_{x}$ leaves the crystal structure invariant, then according to Eq. (1) the tensor $\sigma^{a b c}$ can be nonzero only when $x$ appears an even number of times (zero or two) in $a b c$. This is a phenomenological constraint, which holds at any frequency and irrespective of the mechanism behind the BPVE; it also holds if the space-group operation is not a pure reflection but a glide, in which case the operation $M_{z}$ is still present in the point group. More generally, $\sigma^{a b c}=$ $\sigma^{a c b}$ transforms under point-group operations in the same way as the piezoelectric tensor $d^{a b c}=d^{a c b}$, whose symmetryallowed components have been tabulated for every crystal class [23].

Now assume that the space-group operation is a pure reflection $M_{x}$ and that the minimum direct gap $E_{g}=E_{c}-E_{v}$ is located on an $M_{x}$-invariant plane in the Brillouin zone (BZ). Under these conditions, the shift photoconductivity at frequencies close to $E_{g}$ is further restricted by dipole selection rules, in much the same way as the optical absorption $[25,26]$. The reason is that the states $|v\rangle$ and $|c\rangle$ at the top of the valence band and bottom of the conduction band are now eigenstates of $M_{x}$, with eigenvalues $\pm i$ in the spinful case and \pm 1 in the spinless case; introducing a relative mirror parity $\mathcal{P}_{v c}^{x}$ that equals $+1(-1)$ when $|v\rangle$ and $|c\rangle$ have equal (opposite) eigenvalues, the dipole matrix elements are found to satisfy $[25,26]$

$$
\begin{aligned}
& r_{v c}^{x}=0 \quad \text { when } \mathcal{P}_{v c}^{x}=+1, \\
& r_{v c}^{y}=r_{v c}^{z}=0 \quad \text { when } \mathcal{P}_{v c}^{x}=-1 .
\end{aligned}
$$

By virtue of Eqs. (2) and (3), these selection rules set some components of $\sigma^{a b c}(\omega)$ to zero for $\hbar \omega \approx E_{g}$.

Let us first consider the shift current induced by light linearly polarized along $b=c$. In this case, the phenomenological constraint mentioned above becomes $\sigma^{x b b}=0$; hence the current must flow parallel to the mirror plane. Concerning the band-edge response, one can distinguish two scenarios on the basis of Eq. (4). When $\mathcal{P}_{v c}^{x}=+1$ the matrix element $I_{m n}^{a b b}$ vanishes for $b=x$ so that $\sigma^{a x x}=0$, and when $\mathcal{P}_{v c}^{x}=-1$ it vanishes for $b \neq x$ so that $\sigma^{a y y}=\sigma^{a z z}=0$. Thus, when $\mathcal{P}_{v c}^{x}=+1\left(\mathcal{P}_{v c}^{x}=-1\right)$ the shift current flows along the mirror plane in response to the component of the optical electric field that is parallel (perpendicular) to that plane.

To complete the present discussion, let us consider possible contributions to the shift current from $\sigma^{a b c}$ with $b \neq c$. These average to zero for unpolarized light (e.g., sunlight); hence they are often ignored when discussing solar-cell applications of the BPVE $[5,27,28]$. When they are present, the current is no longer constrained to flow along the mirror plane. At the band edge, $\mathcal{P}_{v c}^{x}=+1$ imposes no restriction at all, while $\mathcal{P}_{v c}^{x}=-1$ forces $\sigma^{a b c}$ to vanish if both $b \neq x$ and $c \neq x$.
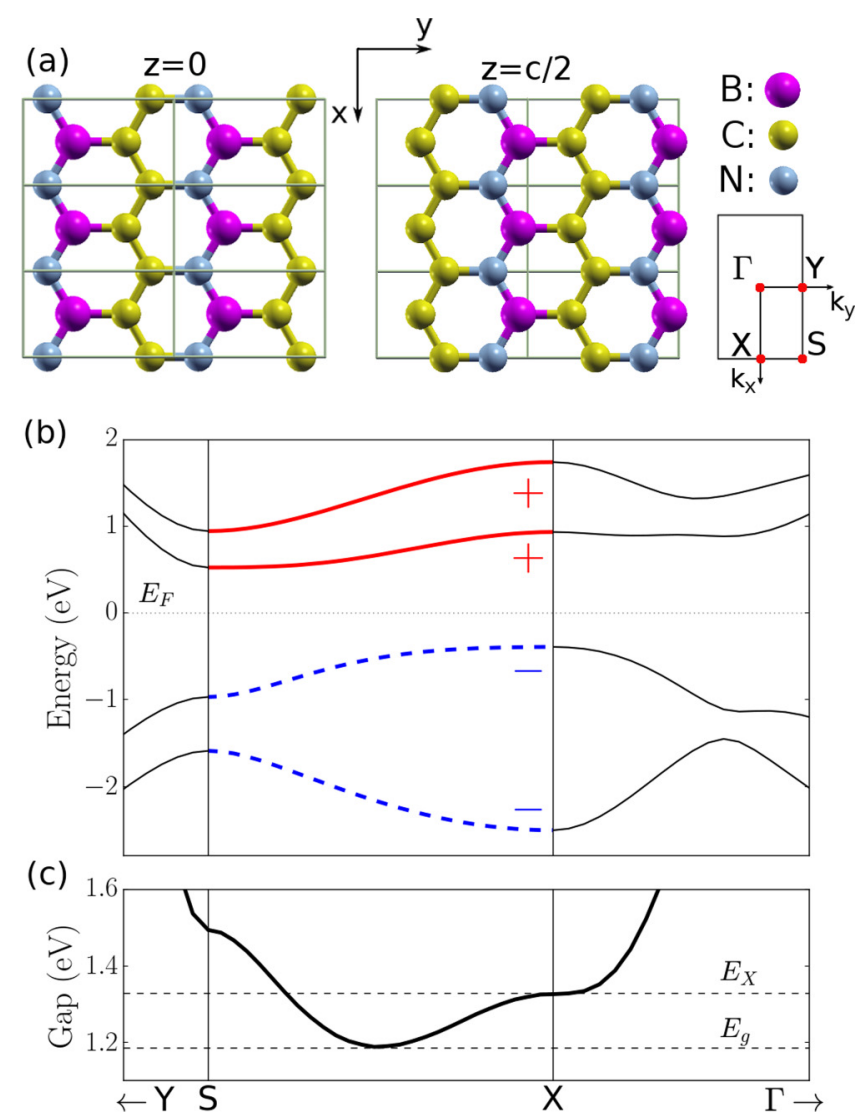

FIG. 1. (a) Two adjacent layers of the $\mathrm{BC}_{2} \mathrm{~N}-\mathrm{A} 2$ structure, with two formula units per cell of height $c$. Solid gray lines delimit the cells (horizontal lines coincide with one family of $M_{x}$ symmetry planes). The $2 \mathrm{D} \mathrm{BZ}$ is also illustrated. (b) Band structure on the $k_{z}=0$ plane. Along $S-X$, thick solid red and dashed blue lines denote bands with $M_{x}$ eigenvalues +1 and -1 , respectively. Along other symmetry lines bands are drawn as thin solid lines. (c) Direct band gap, with values $E_{g}$ and $E_{X}$ at the band edge and $X$, respectively.

\section{PHYSICAL REALIZATION}

We now show that graphitic $\mathrm{BC}_{2} \mathrm{~N}$ provides a striking illustration of the preceding discussion. We begin by noting that while a single layer breaks inversion symmetry [see Fig. 1(a)], whether inversion is still broken in the bulk structure depends on the stacking pattern, which remains to be determined experimentally. There are two types of stackings, denoted by A or B depending on whether consecutive layers have the same or opposite orientation [29]. B-type structures have a center of inversion between the layers, while those of type A break inversion symmetry; hence the photoconductivity remains finite only in A-type structures.

We consider the most stable A-type bulk structure identified in Ref. [29], namely, the A2 structure illustrated in Fig. 1(a). The space group is Pmm2 (No. 25) and the point group is $m m 2$. There are two mirrors $M_{x}$ and $M_{z}$ and a rotation $C_{2}^{y}$ about the polar axis. Point-group symmetry allows 5 out of the 18 independent components of the linear BPVE tensor $\sigma^{a b c}=\sigma^{a c b}$ to be nonzero (the same as for the piezoelectric tensor [23]): three involving in-plane directions only ( $y x x$, $x x y=x y x$, and $y y y)$ and two that also involve $z$ ( $y z z$ and $z z y=z y z)$. Since $M_{x}$ and $M_{z}$ are pure reflections, at the band 
TABLE I. Selection rules for the band-edge shift photoconductivity $\sigma^{a b c}\left(\hbar \omega \approx E_{g}\right)$ in the crystal class $m m 2$. Each column lists one of the symmetry-allowed components, followed by the relative $M_{x}$ and $M_{z}$ band-edge parities that allow that component to be non-negligible for $\hbar \omega \approx E_{g}$. The relative parities for $\mathrm{BC}_{2} \mathrm{~N}-\mathrm{A} 2$, $\mathcal{P}_{v c}^{x}=-1$ and $\mathcal{P}_{v c}^{z}=+1$, are marked in bold; they imply that only $\sigma^{y x x}$ and $\sigma^{x x y}=\sigma^{x y x}$ (also marked in bold) are non-negligible at the band edge.

\begin{tabular}{lccccc}
\hline \hline & \multicolumn{5}{c}{ Components of $\sigma^{a b c}\left(\hbar \omega \approx E_{g}\right)$} \\
\cline { 2 - 6 } Relative parity & $y x x$ & $x x y=x y x$ & $y y y$ & $y z z$ & $z z y=z y z$ \\
\hline $\mathcal{P}_{v c}^{x}$ & $\mathbf{- 1}$ & $+1 /-\mathbf{1}$ & +1 & +1 & +1 \\
$\mathcal{P}_{v c}^{z}$ & $\mathbf{+ 1}$ & $\mathbf{+ 1}$ & $\mathbf{+ 1}$ & -1 & $\mathbf{+ 1} /-1$ \\
\hline \hline
\end{tabular}

edge further restrictions emerge from dipole selection rules as detailed in Table I.

The scalar-relativistic band structure of $\mathrm{BC}_{2} \mathrm{~N}-\mathrm{A} 2$ near the band edge is displayed in Fig. 1(b). We only show the dispersion on the $k_{z}=0$ plane, because the weak interlayer coupling produces a quasi-two-dimensional (quasi-2D) band structure with virtually no $k_{z}$ dispersion [29]. Inspection of the figure reveals that the dispersion from $S$ to $X$ is also relatively weak. The minimum direct band gap of $E_{g} \approx 1.18 \mathrm{eV}$ is located approximately midway between those two timereversal invariant momenta (TRIMs), as shown in Fig. 1(c). On the $S$ - $X$ line, whose points remain invariant under $M_{x}$, the energy eigenstates are also eigenstates of $M_{x}$, with eigenvalues \pm 1 as depicted by the solid and dashed lines in Fig. 1(b). We have explicitly verified [30] that the upper-valence and lower-conduction bands have opposite $M_{x}$ eigenvalues, i.e., $\mathcal{P}_{v c}^{x}=-1$. Moreover, since all bands in Fig. 1(b) are derived from $p_{z}$-type Wannier functions (discussed below), they all have the same $M_{z}$ eigenvalue -1 on the $k_{z}=0$ plane, and hence $\mathcal{P}_{v c}^{z}=+1$. With these two parity values in hand, we predict from Table I that the components of $\sigma^{a b c}$ that are expected to be present at the band edge are $y x x$ and $x x y=x y x$.

We have computed the shift photoconductivity of $\mathrm{BC}_{2} \mathrm{~N}$ A2 by means of density-functional theory (DFT), using the QUANTUM ESPRESSO code package [31]. We took the structural parameters from Ref. [29] and used scalar-relativistic pseudopotentials with the Perdew-BurkeErnzerhof exchange-correlation (XC) functional [32]. Maximally localized Wannier functions $[33,34]$ were generated with the WANNIER90 package [35], starting from atomcentered $p_{z}$ orbitals for modeling the bands around the Fermi level. Finally, the photoconductivity was calculated using a recently developed Wannier-interpolation scheme [36].

The calculated photoconductivity is shown in Fig. 2(a). As predicted in Table I, three of the five independent components that are in principle allowed by point-group symmetry, $\sigma^{y y y}$, $\sigma^{z z y}$ and $\sigma^{y z z}$, have negligible values in the band-edge region indicated by the gray area. The other two, $\sigma^{y x x}$ and $\sigma^{x x y}$, grow rapidly from the onset at $E_{g}$ until reaching peak values of $\sigma^{y x x} \sim 50 \mu \mathrm{AV}^{-2}$ and $\sigma^{x x y} \sim 30 \mu \mathrm{AV}^{-2}$ at $E_{X} \approx 1.33 \mathrm{eV}$; above $E_{X}$ they drop gradually and then stabilize at roughly half their peak values, before peaking again near $2 \mathrm{eV}$ (not shown). The three previously negligible components become sizable above $E_{X}$ due to contributions from valence and

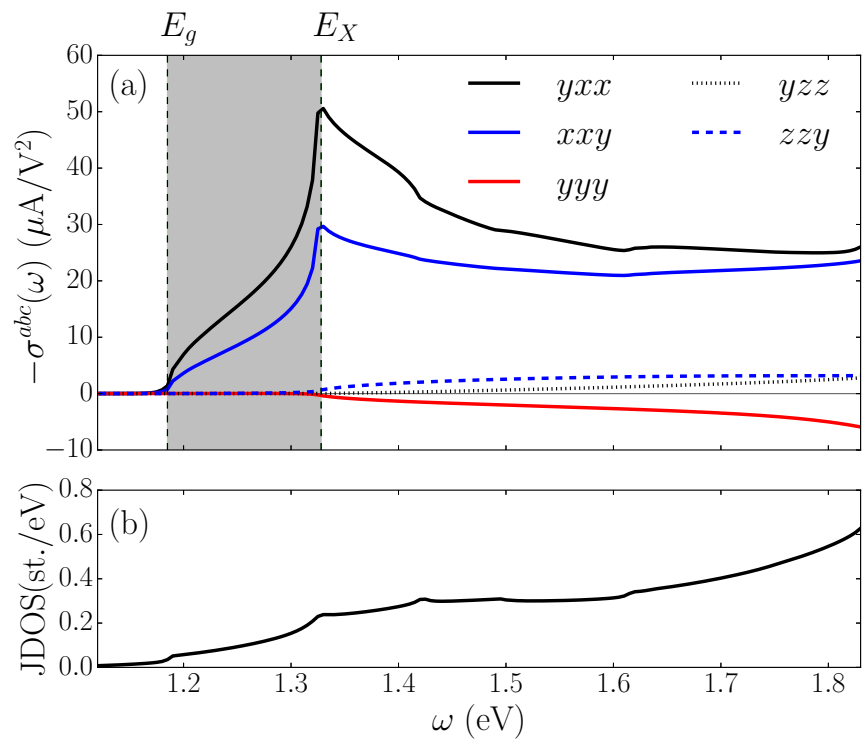

FIG. 2. (a) Shift photoconductivity of bulk $\mathrm{BC}_{2} \mathrm{~N}-\mathrm{A} 2$ calculated $a b$ initio. The gray area indicates the band-edge energy range from $E_{g}$ to $E_{X}$ [see Fig. 1(c)]. (b) Calculated JDOS.

conduction bands outside the mirror-invariant $S$-X line [see Figs. 1(b) and 1(c)], but they remain small compared to the other two.

For light with linear polarization along a crystallographic axis $[b=c$ in Eq. (1)], the spectrum in Fig. 2(a) can be rationalized as follows: At all frequencies $\hbar \omega>E_{g}$ the shift current flows along the line of intersection between the two mirror planes (along $y$ ), and at band-edge frequencies it only flows in response to the field component along $x$ (normal to $M_{x}$ with $P_{v c}^{x}=-1$ and parallel to $M_{z}$ with $\left.P_{v c}^{z}=+1\right)$.

We now turn our attention to the strong peak in $\sigma^{y x x}$ at $E_{X}$. The peak value is rather large for a gapped bulk material; for comparison, among the largest values reported in the literature are $\sim 50 \mu \mathrm{AV}^{-2}$ in ferroelectric $\mathrm{PbTiO}_{3}$ at $6 \mathrm{eV}$ [37] and $\sim 80 \mu \mathrm{AV}^{-2}$ at $1.3 \mathrm{eV}$ in the chiral crystal $\mathrm{RhBiS}$ [38]. The peak photoconductivity in $\mathrm{BC}_{2} \mathrm{~N}$ occurs at a frequency of $\hbar \omega \approx 1.3 \mathrm{eV}$ that is suitable for optical manipulation and where bulk semiconductors typically have much smaller responses $[5,39,40]$. The characteristics of representative photovoltaic materials are collected in Table II, where the last two entries correspond to low-dimensional materials; while their peak photoconductivities surpass that of $\mathrm{BC}_{2} \mathrm{~N}$ $\mathrm{A} 2$, the reported values depend on an adjustable interlayerdistance parameter [6,27]. Furthermore, the 3D crystallization of these low-dimensional structures may not occur in reality, or it may restore inversion symmetry [46]. Free from these concerns, $\mathrm{BC}_{2} \mathrm{~N}$ is a truly bulk material with a large and highly directional photoconductivity in the visible range.

In order to make a more direct connection with potential experimental measurements, it is useful to report two other figures of merit in addition to the photoconductivity: the Glass coefficient [5,47], which quantifies photocurrent generation in bulk materials taking absorption into account, and the shift distance [41] (or anisotropy distance [48]), which gives an estimate of the real-space shift undergone by an electron upon photoexcitation. For light linearly polarized along $b$, the Glass 
TABLE II. Peak shift photoconductivities, peak frequencies, and employed XC functionals, for a collection of bulk and lowdimensional photovoltaic materials. Here GGA denotes generalized gradient approximation and LDA local-density approximation.

\begin{tabular}{lccc}
\hline \hline Material & $\sigma^{a b c}\left(\mu \mathrm{AV}^{-2}\right)$ & $\omega(\mathrm{eV})$ & XC functional \\
\hline $\mathrm{BC}_{2} \mathrm{~N}-\mathrm{A} 2^{\mathrm{a}}$ & 50 & 1.3 & GGA \\
$\mathrm{PbTiO}_{3}, \mathrm{BaTiO}_{3}{ }^{\mathrm{b}}$ & 50,30 & $6.0,6.5$ & GGA \\
$\mathrm{GaAs}^{\mathrm{c}}$ & 40 & 5.5 & $\mathrm{LDA}+$ scissors \\
$\mathrm{LiAsSe}_{2}, \mathrm{NaAsSe}_{2}{ }^{\mathrm{d}}$ & 13,15 & $2.0,3.1$ & $\mathrm{GGA}+$ scissors \\
$\mathrm{BiFeO}_{3}{ }^{\mathrm{c}}$ & 0.8 & 3.5 & GGA $+U$ \\
$\mathrm{RhBiS}_{\mathrm{IrBiSe}}$ & 80,40 & $1.3,2.1$ & GGA \\
$\mathrm{CaAlSiH}^{\mathrm{g}}$ & 6 & 1.3 & GGA \\
$2 \mathrm{D} \mathrm{GeS}, \mathrm{GeSe}^{\mathrm{h}}$ & 160,200 & $2.8,2.0$ & GGA + scissors \\
$1 \mathrm{D} \mathrm{polymers}$ & $60-180$ & $0.6-0.8$ & GGA \\
\hline \hline
\end{tabular}

a This work.

${ }^{\mathrm{b}}$ Reference [37].

${ }^{\mathrm{c}}$ Reference [41].

${ }^{\mathrm{d}}$ Reference [42].

${ }^{\mathrm{e}}$ Reference [43].

${ }^{\mathrm{f}}$ Reference [38].

geference [44].

${ }^{\mathrm{h}}$ Reference [6].

${ }^{\mathrm{i}}$ Reference [45].

coefficient is defined as

$$
G^{a b b}=\frac{1}{2 c \epsilon_{0}} \frac{\sigma^{a b b}(\omega)}{\alpha^{b b}(\omega)},
$$

with $\epsilon_{0}$ the vacuum permittivity, $\alpha^{b b}(\omega)=2 \omega \operatorname{Im} \sqrt{\epsilon^{b b}(\omega)} / c$ the absorption coefficient, and $\epsilon^{b b}(\omega)$ the complex dielectric function. In turn, the shift distance is defined as [41]

$$
d^{a b b}=\frac{\hbar}{e} \frac{\sigma^{a b b}(\omega)}{\operatorname{Im} \epsilon^{b b}(\omega)} .
$$

At frequencies near the band edge where $\operatorname{Im} \epsilon^{b b}(\omega) \ll$ $\operatorname{Re} \epsilon^{b b}(\omega)$, we have $\operatorname{Im} \sqrt{\epsilon^{b b}(\omega)} \simeq \operatorname{Im} \epsilon^{b b}(\omega) / 2$, leading to the relation

$$
G^{a b b} \simeq \frac{e}{2 \epsilon_{0}} \frac{d^{a b b}}{\hbar \omega} .
$$

The quantities $d^{y x x}(\omega)$ and $G^{y x x}(\omega)$ are plotted in Fig. 3. Both spectra are similar to the photoconductivity spectrum in Fig. 2(a), with prominent peaks at $1.3 \mathrm{eV}$. The maximum shift distance is slightly larger than the average bond length between $\mathrm{C}-\mathrm{N}$ and $\mathrm{C}-\mathrm{B}$ distances indicated by the dashed horizontal line at $1.44 \AA$. This means that despite the large shift-current photoconductivity, the real-space shift of the photoelectron is not particularly large. On the other hand, the peak value of the Glass coefficient is $2 \times 10^{-8} \mathrm{~cm} / \mathrm{V}$, which ranks among the largest reported to date [14]. This difference in the relative magnitudes of the two quantities is partly due to the $1 / \omega$ factor in Eq. (7).

To analyze the photoconductivity near the band edge, we approximate it as the product between the shift-current matrix element and the joint density of states (JDOS) [27]

$$
\sigma^{a b c}(\omega) \simeq C I_{v c}^{a b c}(\omega) N(\omega) .
$$

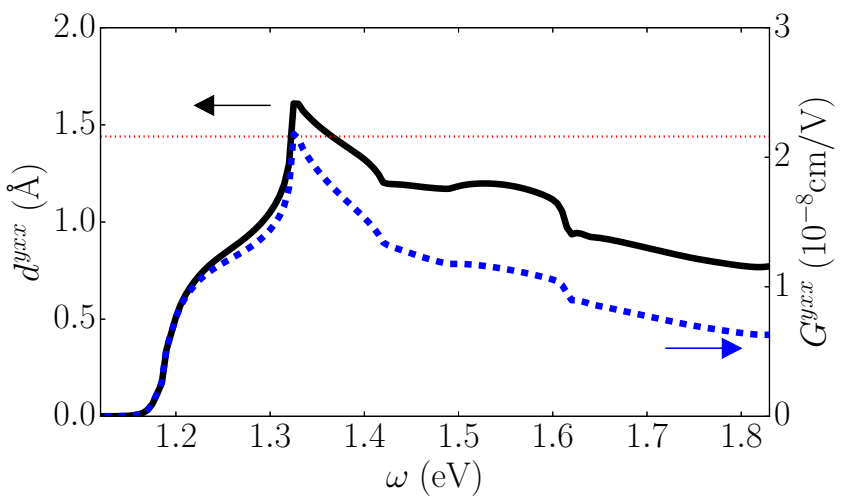

FIG. 3. Solid black and dashed blue lines show the $y x x$ component of the shift distance and Glass coefficient whose corresponding ordinate axes are placed on the left and right of the graph, respectively. The horizontal dotted red line denotes the average bond length between $\mathrm{C}-\mathrm{N}$ and $\mathrm{C}-\mathrm{B}$ distances.

The JDOS, plotted in Fig. 2(b), exhibits a 2D-like Van Hove singularity at $E_{X}$ due to a saddle point in the direct band gap [see Fig. 1(c)], boosting the electronic transitions that contribute to the shift current around that energy. Moreover, those transitions carry a sharply enhanced matrix element for $\sigma^{y x x}$. This can be seen in Fig. 4, which displays a heatmap plot of the matrix element $I_{m n \mathbf{k}}^{y x x}$ of Eq. (2) summed over the upper-valence and lower-conduction bands: Around $X$, it is positive valued and more than two orders of magnitude larger than almost anywhere else in the BZ.

\section{TWO-BAND MODEL IN TWO DIMENSIONS}

Motivated by the quasi-2D nature of graphitic $\mathrm{BC}_{2} \mathrm{~N}-\mathrm{A} 2$, we now construct a minimal $2 \mathrm{D}$ model that captures the mirror-parity effect on the photoconductivity near the band edge. The model lies flat on the $(x, y)$ plane and has both $M_{x}$ and $M_{z}$ symmetries. For simplicity, we assume that the band edge lies at a TRIM on an $M_{x}$-invariant line in the 2D BZ. With these constraints, the most general two-band $\mathbf{k} \cdot \mathbf{p}$ Hamiltonian that can be obtained by expanding up to second

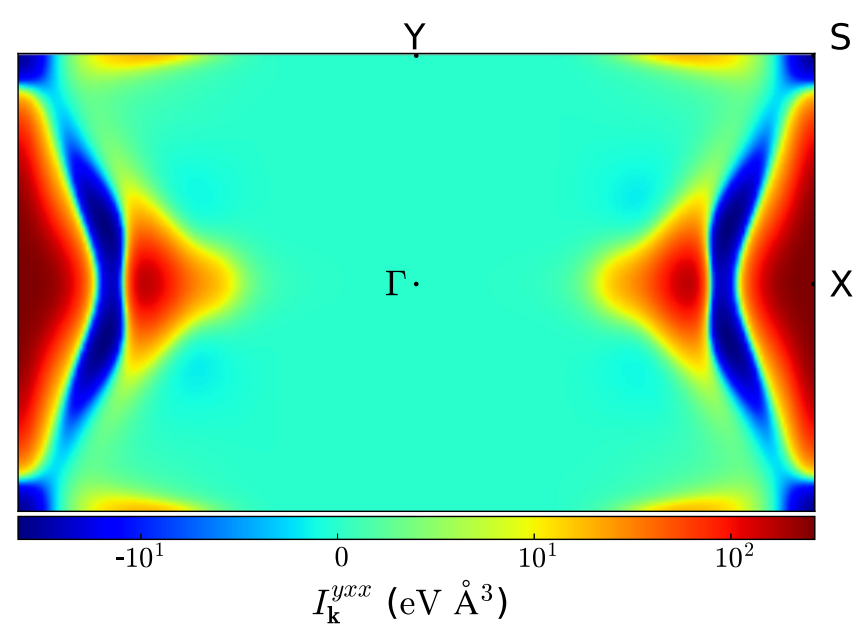

FIG. 4. Heatmap plot across the $2 \mathrm{D} \mathrm{BZ}$ at $k_{z}=0$ of the matrix element $I_{\mathbf{k}}^{y x x}=\sum_{m, n}^{v, c} I_{m n \mathbf{k}}^{y x x}$. 
order in $\mathbf{k}=\left(k_{x}, k_{y}\right)$ around the TRIM is

$$
\begin{aligned}
H\left(k_{x}, k_{y}\right)= & \left(\alpha_{x} k_{x}^{2}+\alpha_{y} k_{y}^{2}+\alpha_{x y} k_{x} k_{y}\right) \sigma_{x} \\
& +\left(v_{x} k_{x}+v_{y} k_{y}\right) \sigma_{y} \\
& +\left(\Delta+\beta_{x} k_{x}^{2}+\beta_{y} k_{y}^{2}+\beta_{x y} k_{x} k_{y}\right) \sigma_{z} .
\end{aligned}
$$

Essentially the same model was considered in Ref. [27], with the following differences: (i) We chose $M_{x}$ as the vertical mirror, which requires keeping terms linear in $k_{x}$ and $k_{y}$, and (ii) we took as basis states the energy eigenstates at the valence and conduction band edges, making our Hamiltonian diagonal at $\mathbf{k}=0$.

Since our basis states are also eigenstates of $M_{x}$ with eigenvalues \pm 1 , the operator $M_{x}$ is represented by the identity matrix when $\mathcal{P}_{v c}^{x}=+1$ and by $\sigma_{z}$ when $\mathcal{P}_{v c}^{x}=-1$. Applying $M_{x} H(\mathbf{k}) M_{x}^{-1}=H\left(M_{x} \mathbf{k}\right)$ to Eq. (9), we find

$$
\begin{aligned}
& v_{x}=\alpha_{x y}=\beta_{x y}=0 \quad \text { when } P_{v c}^{x}=+1, \\
& \alpha_{x}=\alpha_{y}=v_{y}=\beta_{x y}=0 \quad \text { when } P_{v c}^{x}=-1 .
\end{aligned}
$$

The relative band-edge parity $\mathcal{P}_{v c}^{x}$ therefore defines two classes of models with very different properties. The model with $\mathcal{P}_{v c}^{x}=+1$ was used in Ref. [27] to describe the bandedge photoconductivity of monolayer GeS, while the model with $\mathcal{P}_{v c}^{x}=-1$ applies to $\mathrm{BC}_{2} \mathrm{~N}-\mathrm{A} 2$. [Regarding $M_{z}$ symmetry, the model in Eq. (9) has $\mathcal{P}_{v c}^{z}=+1$ because all atomic orbitals have the same parity and lie on the same plane. Hence, $M_{z}$ imposes no further constraints.]

Starting from the two-band Hamiltonian in Eq. (9), the matrix element in Eq. (8) can be evaluated as described in Refs. [27,49]. The nonzero components are

$$
\begin{aligned}
& I_{v c}^{y y y}(\omega)=\frac{8 v_{y} \alpha_{y} \Delta}{\omega^{3}}, \\
& I_{v c}^{x x y}(\omega)=\frac{\left(4 v_{y} \alpha_{x}+2 v_{x} \alpha_{x y}\right) \Delta}{\omega^{3}}=I_{v c}^{x y x}(\omega), \\
& I_{v c}^{y x x}(\omega)=\frac{4 v_{x} \alpha_{x y} \Delta}{\omega^{3}} .
\end{aligned}
$$

Using Eq. (10), we find that when $P_{v c}^{x}=+1$ the $y x x$ component vanishes, while for $P_{v c}^{x}=-1$ it is the yyy component that vanishes. These results are in agreement with the first three columns of Table I for the case $\mathcal{P}_{v c}^{z}=+1$.

Besides illustrating the mirror selection rules, our model reveals a simple quantitative relation

$$
\sigma^{y x x}=2 \sigma^{x x y} \quad \text { when } \mathcal{P}_{v c}^{x}=-1, \quad \mathcal{P}_{v c}^{z}=+1
$$

between the two surviving components of the band-edge photoconductivity. The above relation is satisfied rather well by our $a b$ initio spectrum throughout the entire band-edge region in Fig. 2(a). This can be understood from the fact that Eq. (12) is quite robust: It follows directly from Eq. (3) once we set $\mathcal{P}_{v c}^{x}=-1$ and $\mathcal{P}_{v c}^{z}=+1$ in Eq. (4) and use the identity $r_{c v}^{y ; x}=r_{c v}^{x, y}$ that holds for any two-band tight-binding model once off-diagonal position matrix elements are discarded [36].

\section{DISCUSSION}

To conclude, we discuss the prospects for realizing the physics described herein. The experimental evidence for the stacking sequence in graphitic $\mathrm{BC}_{2} \mathrm{~N}$ remains inconclusive $[16,17]$. According to DFT calculations, the two most stable polytypes are the A2 structure studied in this work and a B-type structure, denoted by B12, with a difference in formation energy of only $1.2 \mathrm{meV} /$ atom favoring the latter [29]. Both are indirect-gap semiconductors, and while B12 provides a slightly better qualitative match to the experimental band structure [29], neither of them fits quantitatively the measured direct and indirect gaps [16,17]. Further work is clearly needed to establish the stacking sequence in bulk $\mathrm{BC}_{2} \mathrm{~N}$ samples, and the linear BPVE could be useful in this regard as it is only present in acentric (A-type) structures.

One intriguing possibility is that it may be possible to grow both polytypes of $\mathrm{BC}_{2} \mathrm{~N}$ using current synthesis techniques. This has been achieved for other layered materials, such as transition metal dichalcogenides [50]. For example, bulk $\mathrm{MoS}_{2}$ grows in two different polytypes, centrosymmetric $2 \mathrm{H}$ and noncentrosymmetric $3 \mathrm{R}$, and the effects of inversion symmetry breaking can be clearly detected in the latter [51]. The reported energy difference between them ranges from 0.1 to $2 \mathrm{meV} /$ atom depending on the calculation [52-54], which is comparable to that between the B12 and A2 structures of $\mathrm{BC}_{2} \mathrm{~N}$ [29]. We hope that our work will stimulate similar progress in graphitic $\mathrm{BC}_{2} \mathrm{~N}$, enabling the unambiguous identification of the $\mathrm{A} 2$ phase via its large and highly anisotropic photogalvanic effect.

\section{ACKNOWLEDGMENTS}

We thank Stepan S. Tsirkin for sharing the IRREP computer code that determines the mirror eigenvalues of the Bloch states [30] and for a previous collaboration on related work. This work was supported by Grant No. FIS2016-77188-P from the Spanish Ministerio de Economía y Competitividad. This project received funding from the European Union's Horizon 2020 research and innovation program under the Marie Sklodowska-Curie Grant Agreement No. 839237.
[1] V. I. Belinicher and B. I. Sturman, The photogalvanic effect in media lacking a center of symmetry, Sov. Phys. Usp. 23, 199 (1980).

[2] B. I. Sturman and V. M. Fridkin, The Photovoltaic and Photorefractive Effects in Noncentrosymmetric Materials (Gordon and Breach, New York, 1992).

[3] V. M. Fridkin, Bulk photovoltaic effect in noncentrosymmetric crystals, Crystallogr. Rep. 46, 654 (2001).
[4] J. Ma, Q. Gu, Y. Liu, J. Lai, P. Yu, X. Zhuo, Z. Liu, J.-H. Chen, J. Feng, and D. Sun, Nonlinear photoresponse of type-II Weyl semimetals, Nat. Mater. 18, 476 (2019).

[5] L. Z. Tan, F. Zheng, S. M. Young, F. Wang, S. Liu, and A. M. Rappe, Shift current bulk photovoltaic effect in polar materials-Hybrid and oxide perovskites and beyond, npj Comput. Mater. 2, 16026 (2016). 
[6] T. Rangel, B. M. Fregoso, B. S. Mendoza, T. Morimoto, J. E. Moore, and J. B. Neaton, Large Bulk Photovoltaic Effect and Spontaneous Polarization of Single-Layer Monochalcogenides, Phys. Rev. Lett. 119, 067402 (2017).

[7] K. Kushnir, M. Wang, P. D. Fitzgerald, K. J. Koski, and L. V. Titova, Ultrafast zero-bias photocurrent in GeS nanosheets: Promise for photovoltaics, ACS Energy Lett. 2, 1429 (2017).

[8] K. Kushnir, Y. Qin, Y. Shen, G. Li, B. M. Fregoso, S. Tongay, and L. V. Titova, Ultrafast zero-bias surface photocurrent in germanium selenide: Promise for terahertz devices and photovoltaics, ACS Appl. Mater. Int. 11, 5492 (2019).

[9] T. Morimoto and N. Nagaosa, Topological nature of nonlinear optical effects in solids, Sci. Adv. 2, e1501524 (2016).

[10] B. M. Fregoso, T. Morimoto, and J. E. Moore, Quantitative relationship between polarization differences and the zoneaveraged shift photocurrent, Phys. Rev. B 96, 075421 (2017).

[11] F. de Juan, A. G. Grushin, T. Morimoto, and J. E. Moore, Quantized circular photogalvanic effect in Weyl semimetals, Nat. Commun. 8, 15995 (2017).

[12] L. Z. Tan and A. M. Rappe, Enhancement of the Bulk Photovoltaic Effect in Topological Insulators, Phys. Rev. Lett. 116, 237402 (2016).

[13] Z. Yan, Precise determination of critical points of topological phase transitions via shift current in two-dimensional inversion asymmetric insulators, arXiv:1812.02191.

[14] G. B. Osterhoudt, L. K. Diebel, M. J. Gray, X. Yang, J. Stanco, X. Huang, B. Shen, N. Ni, P. J. W. Moll, Y. Ran, and K. S. Burch, Colossal mid-infrared bulk photovoltaic effect in a typeI Weyl semimetal, Nat. Mater. 18, 471 (2019).

[15] A. Y. Liu, R. M. Wentzcovitch, and M. L. Cohen, Atomic arrangement and electronic structure of $\mathrm{BC}_{2} \mathrm{~N}$, Phys. Rev. B 39, 1760 (1989).

[16] M. O. Watanabe, S. Itoh, T. Sasaki, and K. Mizushima, VisibleLight-Emitting Layered $\mathrm{BC}_{2} \mathrm{~N}$ Semiconductor, Phys. Rev. Lett. 77, 187 (1996).

[17] Y. Chen, J. C. Barnard, R. E. Palmer, M. O. Watanabe, and T. Sasaki, Indirect Band Gap of Light-Emitting $\mathrm{BC}_{2} \mathrm{~N}$, Phys. Rev. Lett. 83, 2406 (1999).

[18] M. O. Watanabe, T. Sasaki, S. Itoh, and K. Mizushima, Structural and electrical characterization of $\mathrm{BC}_{2} \mathrm{~N}$ thin films, Thin Solid Films 281, 334 (1996).

[19] M. O. Watanabe, S. Itoh, K. Mizushima, and T. Sasaki, Electrical properties of $\mathrm{BC}_{2} \mathrm{~N}$ thin films prepared by chemical vapor deposition, J. Appl. Phys. 78, 2880 (1995).

[20] M. O. Watanabe, S. Itoh, K. Mizushima, and T. Sasaki, Bonding characterization of $\mathrm{BC}_{2} \mathrm{~N}$ thin films, Appl. Phys. Lett. 68, 2962 (1996).

[21] H. Nozaki and S. Itoh, Lattice dynamics of $\mathrm{BC}_{2} \mathrm{~N}$, Phys. Rev. B 53, 14161 (1996).

[22] J. E. Sipe and A. I. Shkrebtii, Second-order optical response in semiconductors, Phys. Rev. B 61, 5337 (2000).

[23] J. F. Nye, Physical Properties of Crystals (Clarendon, Oxford, 1957).

[24] R. E. Newnham, Properties of Materials (Oxford University Press, Oxford, 2005).

[25] G. F. Bassani and G. P. Parravicini, Band structure and optical properties of graphite and of the layer compounds $\mathrm{GaS}$ and GaSe, Nuovo Cimento B 50, 95 (1967).

[26] G. F. Bassani and G. P. Parravicini, Electronic States and Optical Transitions in Solids (Pergamon, Oxford, 1975).
[27] A. M. Cook, B. M. Fregoso, F. de Juan, S. Coh, and J. E. Moore, Design principles for shift current photovoltaics, Nat. Commun. 8, 14176 (2017).

[28] C. Wang, X. Liu, L. Kang, B.-L. Gu, Y. Xu, and W. Duan, Firstprinciples calculation of nonlinear optical responses by Wannier interpolation, Phys. Rev. B 96, 115147 (2017).

[29] Z. Pan, H. Sun, and C. Chen, Interlayer stacking and nature of the electronic band gap in graphitic $\mathrm{BC}_{2} \mathrm{~N}$ : First-principles pseudopotential calculations, Phys. Rev. B 73, 193304 (2006).

[30] S. S. Tsirkin, https://github.com/stepan-tsirkin/irrep.

[31] P. Giannozzi, S. Baroni, N. Bonini, M. Calandra, R. Car, C. Cavazzoni, D. Ceresoli, G. L. Chiarotti, M. Cococcioni, I. Dabo et al., QUANTUM ESPRESSO: A modular and open-source software project for quantum simulations of materials, J. Phys.: Condens. Matter 21, 395502 (2009).

[32] J. P. Perdew, K. Burke, and M. Ernzerhof, Generalized Gradient Approximation Made Simple, Phys. Rev. Lett. 77, 3865 (1996).

[33] N. Marzari and D. Vanderbilt, Maximally localized generalized Wannier functions for composite energy bands, Phys. Rev. B 56, 12847 (1997).

[34] I. Souza, N. Marzari, and D. Vanderbilt, Maximally localized Wannier functions for entangled energy bands, Phys. Rev. B 65, 035109 (2001).

[35] A. A. Mostofi, J. R. Yates, Y.-S. Lee, I. Souza, D. Vanderbilt, and N. Marzari, wannier90: A tool for obtaining maximallylocalised Wannier functions, Comput. Phys. Commun. 178, 685 (2008).

[36] J. Ibañez-Azpiroz, S. S. Tsirkin, and I. Souza, Ab initio calculation of the shift photocurrent by Wannier interpolation, Phys. Rev. B 97, 245143 (2018).

[37] S. M. Young and A. M. Rappe, First Principles Calculation of the Shift Current Photovoltaic Effect in Ferroelectrics, Phys. Rev. Lett. 109, 116601 (2012).

[38] Y. Zhang, F. de Juan, A. G. Grushin, C. Felser, and Y. Sun, Strong bulk photovoltaic effect in chiral crystals in the visible spectrum, Phys. Rev. B 100, 245206 (2019).

[39] S. Y. Yang, J. Seidel, S. J. Byrnes, P. Shafer, C.-H. Yang, M. D. Rossell, P. Yu, Y.-H. Chu, J. F. Scott, J. W. Ager III, L. W. Martin, and R. Ramesh, Above-bandgap voltages from ferroelectric photovoltaic devices, Nat. Nanotechnol. 5, 143 (2010).

[40] W. Ji, K. Yao, and Y. C. Liang, Evidence of bulk photovoltaic effect and large tensor coefficient in ferroelectric $\mathrm{BiFeO}_{3}$ thin films, Phys. Rev. B 84, 094115 (2011).

[41] F. Nastos and J. E. Sipe, Optical rectification and shift currents in GaAs and GaP response: Below and above the band gap, Phys. Rev. B 74, 035201 (2006).

[42] J. A Brehm, S. M Young, F. Zheng, and A. M Rappe, Firstprinciples calculation of the bulk photovoltaic effect in the polar compounds $\mathrm{LiAsS}_{2}, \mathrm{LiAsSe}_{2}$, and $\mathrm{NaAsSe}_{2}$, J. Chem. Phys. 141, 204704 (2014).

[43] S. M. Young, F. Zheng, and A. M. Rappe, First-Principles Calculation of the Bulk Photovoltaic Effect in Bismuth Ferrite, Phys. Rev. Lett. 109, 236601 (2012).

[44] J. A. Brehm, Predicted bulk photovoltaic effect in hydrogenated Zintl compounds, J. Mater. Chem. C 6, 1470 (2018).

[45] S. Liu, F. Zheng, and A. M. Rappe, Giant bulk photovoltaic effect in vinylene-linked hybrid heterocyclic polymer, J. Phys. Chem. C 121, 6500 (2017). 
[46] A. M. Elkorashy, Indirect forbidden fundamental absorption edge in germanium selenide single crystals, Phys. Status Solid B 135, 707 (1986).

[47] A. M. Glass, D. von der Linde, and T. J. Negran, High-voltage bulk photovoltaic effect and the photorefractive process in $\mathrm{LiNbO}_{3}$, Appl. Phys. Lett. 25, 233 (1974).

[48] R. von Baltz and W. Kraut, Theory of the bulk photovoltaic effect in pure crystals, Phys. Rev. B 23, 5590 (1981).

[49] X. Yang, K. Burch, and Y. Ran, Divergent bulk photovoltaic effect in Weyl semimetals, arXiv:1712.09363.

[50] J. A. Wilson and A. D. Yoffe, The transition metal dichalcogenides discussion and interpretation of the observed optical, electrical and structural properties, Adv. Phys. 18, 193 (1969).

[51] R. Suzuki, M. Sakano, Y. J. Zhang, R. Akashi, D. Morikawa, A. Harasawa, K. Yaji, K. Kuroda, K. Miyamoto, T. Okuda,
K. Ishizaka, R. Arita, and Y. Iwasa, Valley-dependent spin polarization in bulk $\mathrm{MoS}_{2}$ with broken inversion symmetry, Nat. Nanotechnol. 9, 611 (2014).

[52] Y. Cheng, K. Yao, Y. Yang, L. Li, Y. Yao, Q. Wang, X. Zhang, Y. Han, and U. Schwingenschlögl, Van der Waals epitaxial growth of $\mathrm{MoS}_{2}$ on $\mathrm{SiO}_{2} / \mathrm{Si}$ by chemical vapor deposition, RSC Adv. 3, 17287 (2013).

[53] J. Suh, T. L. Tan, W. Zhao, J. Park, D.-Y. Lin, T.-E. Park, J. Kim, C. Jin, N. Saigal, S. Ghosh, Z. M. Wong, Y. Chen, F. Wang, W. Walukiewicz, G. Eda, and J. Wu, Reconfiguring crystal and electronic structures of $\mathrm{MoS}_{2}$ by substitutional doping, Nat. Commun. 9, 199 (2018).

[54] X. B. Chen, Z. L. Chen, and J. Li, Critical electronic structures controlling phase transitions induced by lithium ion intercalation in molybdenum disulphide, Chin. Sci. Bull. 58, 1632 (2013). 